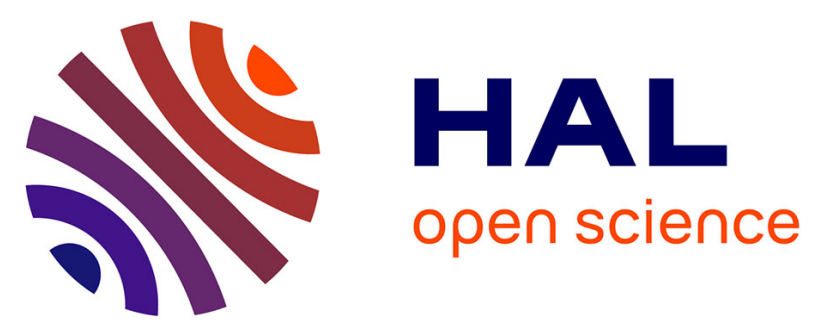

\title{
IMPACT OF TRANSFORMER AND CABLE AGING ON DISTRIBUTION LOCATIONAL MARGINAL COSTS IN ACTIVE DISTRIBUTION NETWORKS
}

\author{
Panagiotis Andrianesis, Andrea Michiorri, Georges Kariniotakis, Michael
}

Caramanis

\section{To cite this version:}

Panagiotis Andrianesis, Andrea Michiorri, Georges Kariniotakis, Michael Caramanis. IMPACT OF TRANSFORMER AND CABLE AGING ON DISTRIBUTION LOCATIONAL MARGINAL COSTS IN ACTIVE DISTRIBUTION NETWORKS. CIRED 2021 Conference, Sep 2021, Online, France. hal-03407221

\section{HAL Id: hal-03407221 \\ https://hal.science/hal-03407221}

Submitted on 28 Oct 2021

HAL is a multi-disciplinary open access archive for the deposit and dissemination of scientific research documents, whether they are published or not. The documents may come from teaching and research institutions in France or abroad, or from public or private research centers.
L'archive ouverte pluridisciplinaire HAL, est destinée au dépôt et à la diffusion de documents scientifiques de niveau recherche, publiés ou non, émanant des établissements d'enseignement et de recherche français ou étrangers, des laboratoires publics ou privés. 


\title{
IMPACT OF TRANSFORMER AND CABLE AGING ON DISTRIBUTION LOCATIONAL MARGINAL COSTS IN ACTIVE DISTRIBUTION NETWORKS Panagiotis Andrianesis $^{1^{*}}$, Andrea Michiorri' ${ }^{2}$, George Kariniotakis ${ }^{2}$, Michael Caramanis ${ }^{3}$
}

\author{
${ }^{1}$ Institute for Sustainable Energy, Boston University, Boston, MA \\ ${ }^{2}$ MINES ParisTech, PSL University, Centre PERSEE, Sophia-Antipolis, France \\ ${ }^{3}$ Mechanical Engineering Department, Boston University, Boston, MA \\ *panosa@bu.edu
}

Keywords: CABLE/TRANSFORMER AGING, DISTRIBUTION NETWORK MARGINAL COSTS, AC OPF.

\begin{abstract}
This paper considers distribution network asset degradation, namely transformer and cable aging, in the context of the day-ahead operational planning in active distribution networks. The focus of this work is to quantify aging as a short-run network variable cost, and complement accordingly Distribution Locational Marginal Cost (DLMC) components. Short-run dynamic DLMCs are defined using sensitivity analysis of a centralized enhanced AC Optimal Power Flow model, providing incentives for optimal DER scheduling. Results indicate that optimal DER scheduling leads to smoother DLMC marginal aging component profiles compared to popular "open-loop", e.g., Time of Use, pricing schemes.
\end{abstract}

\section{Introduction}

Rapid integration of Distributed Energy Resources (DERs), including Electric Vehicles (EVs), solar photovoltaic (PV), and the like, presents several challenges to distribution networks and their assets. For instance, early studies on EVs pointed out that clustering EV chargers under the same transformer may cause damage and outages from persistent overloading.

In a recent work [1], we presented a novel centralized dayahead AC Optimal Power Flow (OPF) model encompassing transformer degradation/aging [2,3] as a short-run network variable cost, and additive real/reactive power Distribution Locational Marginal Cost (DLMC) components related to: the costs of real and reactive power transactions at the T\&D interface; real and reactive power marginal losses; voltage and ampacity congestion, and a transformer degradation/aging marginal cost component. In this work, we further explore cable aging [4, 5], using the cable thermal stress model, which exhibits direct analogies to the transformer thermal model.

Part of our analysis focuses on the incentives provided by the short-run dynamic DLMCs viewed as price signals (see e.g., $[1,6]$, compared with other "open-loop" approaches, e.g., Time of Use (ToU) tariffs. Preliminary results suggest that highly volatile DLMCs obtained by open-loop DER scheduling options tend to become smoother when DERs adapt their profiles to real and reactive power DLMCs [6].

The main contributions are three-fold. First, we complement the enhanced AC OPF model of [1] considering the cost of cable aging. Second, we present a new cable marginal aging DLMC component. Third, we estimate the marginal aging components on an instance of an actual distribution feeder.
The remainder of this paper is organized as follows. Section 2 introduces the cost of aging into the AC OPF model. Section 3 presents the marginal aging DLMC components, and Section 4 estimates them for different load profiles. Section 5 concludes and provides further research directions.

\section{AC OPF Model including Asset Aging}

\subsection{Branch Flow AC OPF Model}

We consider a radial network with $N+1$ nodes, $N$ lines, where $\mathcal{N}=\{0,1, \ldots, N\}$ is the set of nodes, with node 0 representing the root node. The set $\mathcal{N}^{+}=\mathcal{N} \backslash\{0\}$ is also used to denote the set of lines, since each line $i j$, linking upstream node $i$ and downstream node $j$ is uniquely defined by the downstream node $j$. Transformers and cables are represented as subsets of lines by $\mathcal{N}_{X f}^{+}$and $\mathcal{N}_{C}^{+}$, respectively; they are indexed by $j$. Also let $t$ denote the time period, and $T$ the length of the optimization horizon, with $\mathcal{T}^{+}=\{1,2, \ldots T\}$; unless otherwise mentioned $t \in \mathcal{T}^{+}$. For each node $i \in \mathcal{N}$, let $v_{i, t}$ denote the magnitude squared voltage, $p_{i, t}\left(q_{i, t}\right)$ denote the net demand of real (reactive) power. For each line $j \in \mathcal{N}^{+}$, let $l_{j, t}$ denote the magnitude squared current, $P_{j, t}\left(Q_{j, t}\right)$ the real (reactive) power flow, and $r_{j}\left(x_{j}\right)$ the resistance (reactance).

The AC OPF model objective function:

$$
\operatorname{minimize} \sum_{t} c_{t}^{P} P_{1, t}+\sum_{t} c^{Q} Q_{1, t}+\text { Aging Cost }
$$

minimizes the sum of the real power cost, where $c_{t}^{P}$ is the hourly cost of real power at the root node, plus the the sum of the reactive power cost, where $c_{t}^{Q}$ is an opportunity cost for reactive power at the root node, plus the asset aging cost. In 
(1), we assumed, without loss of generality that the root node is only connected to line 1 , hence the net injections at the root node, equal the flows $P_{1, t}$ and $Q_{1, t}$.

The branch flow model [7] describes the power flow equations as follows:

$$
\begin{gathered}
P_{j, t}-r_{j} l_{j, t}=\sum_{j^{\prime}: j \rightarrow j^{\prime}} P_{j^{\prime}, t}+p_{j, t}, \quad \forall j, t, \\
Q_{j, t}-x_{j} l_{j, t}=\sum_{j^{\prime}: j \rightarrow j^{\prime}} Q_{j^{\prime}, t}+q_{j, t}, \quad \forall j, t, \\
v_{j, t}=v_{i, t}-2 r_{j} P_{j, t}-2 x_{j} Q_{j, t}+\left(r_{j}^{2}+x_{j}^{2}\right) l_{j, t}, \quad \forall j, t, \\
v_{i, t} l_{j, t}=P_{j, t}^{2}+Q_{j, t}^{2}, \quad \forall j, t
\end{gathered}
$$

where (2) and (3) define the real and reactive power balance at node $j$, respectively, (4) defines the voltage drop along line $j$, and (5) defines the apparent power flow along line $j$. The latter non-convex equality constraint is relaxed to a convex inequality $v_{i, t} l_{j, t} \geq P_{j, t}^{2}+Q_{j, t}^{2}$, following [8], yielding a Second Order Cone Programming (SOCP) relaxation.

\subsection{Transformer Aging and Thermal Model}

In [1], we considered the transformer Loss of Life (LoL) using the Arrhenius model for the insulation relative aging or Aging Acceleration Factor (AAF) [2, 3], given by:

$$
\mathrm{AAF}_{j, t}=\exp \left(\frac{A}{383}-\frac{A}{273+\theta_{j, t}^{H}}\right),
$$

where $A$ is a constant with typical value 15000 , and $\theta_{j, t}^{H}$ is the transformer Hottest Spot Temperature (HST). The AAF is equal to one for a reference $\mathrm{HST}$ of $=110^{\circ} \mathrm{C}$, whereas it is greater (lower) than 1 for higher (lower) HSTs.

The transformer aging cost is defined as:

$$
\text { Transformer Aging Cost }=\sum_{j \in \mathcal{N}_{X f}^{+}, t} c_{j} L_{j, t},
$$

with $c_{j}$ an hourly cost that is obtained considering its value and estimated lifetime, and $L_{j, t}$ a piecewise linearization, with $M$ segments, of the transformer LoL that is given by:

$$
L_{j, t} \geq a_{m}^{H} \theta_{j, t}^{H}-b_{m}^{H}, \quad m=1, \ldots, M,
$$

where the relaxed inequality is guaranteed to be exact, since $L_{j, t}$ is introduced with a cost in the objective function.

The HST is given by:

$$
\theta_{j, t}^{H}=\theta_{t}^{A}+\Delta \theta_{j, t}^{T O}+\Delta \theta_{j, t}^{H}=\theta_{j, t}^{T O}+\Delta \theta_{j, t}^{H},
$$

where $\theta_{t}^{A}$ is the Ambient (A) temperature, $\Delta \theta_{j, t}^{T O}$ is the TopOil (TO) temperature rise over $\theta_{t}^{A}$, and $\Delta \theta_{j, t}^{H}$ is the winding HST temperature rise over $\theta_{j, t}^{T O}$. The temperature dynamics of the transformer thermal response include a fast response of the winding with a time constant of about 3-4 minutes, and a slower response of the top-oil, with a time constant of about 3 hours.
Hence, assuming an operational-planning scheduling horizon with a time granularity of $15 \mathrm{~min}$ to $1 \mathrm{~h}$, we can account for the temperature dynamics of the transformer, by considering the steady state of the winding, and the difference equations for the top-oil temperature. The detailed derivations are provided in [1] and are summarized, $\forall j \in \mathcal{N}_{X f}^{+}, t, m=1, \ldots, M$, in:

$$
\begin{gathered}
L_{j, t} \geq \alpha_{m}^{H} \theta_{j, t}^{T O}+\beta_{j, m}^{H} l_{j, t}+\gamma_{j, m}^{H}, \\
\theta_{j, t}^{T O}=\delta^{T O} \theta_{j, t-1}^{T O}+\epsilon_{j}^{T O} l_{j, t}+\zeta_{j, t}^{T O} .
\end{gathered}
$$

Using an hourly granularity, recommended values for service transformers, and a typical ratio of load losses at rated load to no-load losses equal to 5, the parameter values are given as follows: $\alpha_{m}^{H}=a_{m}^{H}, \beta_{j, m}^{H}=\frac{0.8 a_{m}^{H} \Delta \bar{\theta}_{j}^{H}}{l_{j}^{R}}$, $\gamma_{j, m}^{H}=0.2 a_{m}^{H} \Delta \bar{\theta}_{j}^{H}-b_{m}^{H} . \delta^{T O}=\frac{3}{4}, \epsilon_{j}^{T O}=\frac{0.167 \Delta \bar{\theta}_{j}^{T O}}{l_{j}^{R}}, \zeta_{j, t}^{T O}=$ $0.083 \Delta \bar{\theta}_{j}^{T O}+0.25 \theta_{t}^{A}$, where $l_{j}^{R}$ represents the transformer rated magnitude squared current, whereas $\Delta \bar{\theta}_{j}^{T O}$ and $\Delta \bar{\theta}_{j}^{H}$ represent the top-oil and winding temperature rise at rated load, with typical values of $55^{\circ} \mathrm{C}$ and $25^{\circ} \mathrm{C}$, respectively. For completeness, the transformer HST is given by:

$$
\theta_{j, t}^{H}=\theta_{j, t}^{T O}+0.8 \Delta \bar{\theta}_{j}^{H} \frac{l_{j, t}}{l_{j}^{R}}+0.2 \Delta \bar{\theta}_{j}^{H} .
$$

\subsection{Cable Aging and Thermal Model}

The insulation of electric cables is also subject to aging following the Arrhenius model. For a given temperature of conductor core $j$, at time period $t, \theta_{j, t}^{C}$, the AAF is given by:

$$
\mathrm{AAF}_{j, t}=\exp \left(\frac{B}{363}-\frac{B}{273+\theta_{j, t}^{C}}\right)
$$

where $B$ is a constant that depends on the cable. The AAF indicates that, at a reference conductor core temperature of $90^{\circ} \mathrm{C}$, the cable loses one hour of life for one hour operation. The cable AAF is greater (lower) than 1 for conductor core temperatures greater (lower) than $90^{\circ} \mathrm{C}$.

The thermal modeling of the conductor is based on IEC standards $[4,5]$, which describe power cable rating calculations in steady state and dynamic conditions. The conductor core temperature, $\theta_{j, t}^{C}$, is given by:

$$
\theta_{j, t}^{C}=\theta_{t}^{S}+\Delta \theta_{j, t}^{C S}+\Delta \theta_{j, t}^{C}=\theta_{j, t}^{C S}+\Delta \theta_{j, t}^{C},
$$

where $\theta_{t}^{S}$ is the temperature of the Soil (S) far from the cable (environment temperature), $\Delta \theta_{j, t}^{C S}$ is the Cable Surface (CS) temperature rise over $\theta_{t}^{S}$, and $\Delta \theta_{j, t}^{C}$ is the conductor Core (C) temperature rise over $\theta_{j, t}^{C S}$. Note the similarity of (14) with (9); indeed the soil temperature resembles the ambient temperature, the cable surface temperature (rise) resembles the top-oil temperature (rise), and the conductor core temperature (rise) resembles the winding HST temperature (rise).

For the needs of our analysis that considers the operationalplanning problem time granularity, we distinguish between the two thermal responses, which describe $\Delta \theta_{j, t}^{C S}$, and $\Delta \theta_{j, t}^{C}$. The 
thermal time constant of the conductor (order of a few minutes) allows us to model $\Delta \theta_{j, t}^{C}$ using its steady state, i.e.,

$$
\Delta \theta_{j, t}^{C}=\Delta \bar{\theta}_{j}^{C} \frac{l_{j, t}}{l_{i}^{R}}
$$

where $\Delta \bar{\theta}_{j}^{C}$ is the conductor core temperature rise above the cable surface temperature at rated current, and $l_{j}^{R}$ is the conductor rated magnitude squared current. The thermal model for the cable surface temperature has a time constant, $\tau^{S}$, of about $2 \mathrm{~h}$. Since the time granularity (of at most $1 \mathrm{~h}$ ) is at least half the time constant, the cable surface temperature dynamics can be described by difference equations:

$$
\Delta \bar{\theta}_{j}^{C S} \frac{l_{j, t}}{l_{j}^{R}}=\tau^{s} \frac{\theta_{j, t}^{C S}-\theta_{j, t-1}^{C S}}{\Delta t}+\theta_{j, t}^{C S}-\theta_{t}^{S},
$$

where $\Delta \bar{\theta}_{j}^{C S}$ is the cable surface temperature rise above the soil temperature at rated current, and $\Delta t$ is the time granularity. Hence, from (16), setting $\delta^{S}=\frac{\tau^{S}}{\tau^{S}+\Delta t}$, we get:

$$
\theta_{j, t}^{C S}=\delta^{S} \theta_{j, t-1}^{C S}+\frac{\left(1-\delta^{S}\right) \Delta \bar{\theta}_{j}^{C S}}{l_{j}^{R}} l_{j, t}+\left(1-\delta^{S}\right) \theta_{t}^{S} .
$$

Similarly to the transformer, the cable aging cost is given by:

$$
\text { Cable Aging Cost }=\sum_{j \in \mathcal{N}_{X f}^{+}, t} c_{j} L_{j, t},
$$

where $c_{j}$ is an hourly cost that considers the cable value and estimated lifetime, and $L_{j, t}$ is a piecewise linearization of the AAF, with with $M$ segments, similarly to (8),

$$
L_{j, t} \geq a_{m}^{C} \theta_{j, t}^{C}-b_{m}^{C}, \quad m=1, \ldots, M .
$$

Using (15) and (16), we replace $\theta_{j, t}^{C}$ from (14) to (19), to get, $\forall j \in \mathcal{N}_{C}^{+}, t, m=1, \ldots, M$ :

$$
\begin{gathered}
L_{j, t} \geq \alpha_{m}^{C} \theta_{j, t}^{C S}+\beta_{j, m}^{C} l_{j, t}+\gamma_{j, m}^{C}, \\
\theta_{j, t}^{C S}=\delta^{S} \theta_{j, t-1}^{C S}+\epsilon_{j}^{C S} l_{j, t}+\zeta_{t}^{S} .
\end{gathered}
$$

Using $\Delta t=1 \mathrm{~h}$ and $\tau^{S}=2 \mathrm{~h}$, the parameter values are given as follows: $\alpha_{m}^{C}=a_{m}^{C}, \beta_{j, m}^{C}=\frac{a_{m}^{C} \Delta \bar{\theta}_{j}^{C}}{l_{j}^{R}}, \gamma_{j, m}^{C}=-b_{m}^{C} \cdot \delta^{S}=\frac{2}{3}$, $\epsilon_{j}^{C S}=\frac{\Delta \bar{\theta}_{j}^{C S}}{3 l_{j}^{R}}, \zeta_{t}^{S}=\frac{\theta_{t}^{S}}{3}$. Typical values for $\Delta \bar{\theta}_{j}^{C S}$ and $\Delta \bar{\theta}_{j}^{C}$ are $38^{\circ} \mathrm{C}$ and $40^{\circ} \mathrm{C}$; they should be calibrated to the specific cable. For the calculations, we neglected cable dielectric losses and the impact of soil moisture, soil thermal resistivity was set to $1.2[\mathrm{WK} / \mathrm{m}]$ and the soil temperature was set to $12^{\circ} \mathrm{C}$. The typical values also depend on the type of the conductor, and their installation characteristics, e.g., conductors directly buried in the ground as opposed to conductors laid in ducts or galleries.

\subsection{Operational-Planning Problem Summary}

The objective function includes (1), where the Aging Cost is given by (7) and (18). Constraints include: Power flow constraints (2)-(5), plus transformer aging constraints (10)-(11), plus cable aging constraints (20)-(21), plus nodal voltage and ampacity limits, plus DER constraints and preferences that detail nodal demand; we refer the interested reader to [1] for a detailed description of the constraints not included herein due to space limitations. The optimization problem is a convex SOCP problem, which can be solved with commercially available solvers.

\section{Distribution Locational Marginal Costs}

DLMCs for real and reactive power, denoted by P-DLMCs and Q-DLMCs, are obtained by the dual variables of constraints (2) and (3), denoted by $\lambda_{j, t}^{P}$ and $\lambda_{j, t}^{Q}$, respectively. Equivalently, the DLMCs can be also calculated employing sensitivity analysis, duality and optimality conditions for a given operating point of the network, which is in turn determined by the solution of the power flow equations, for a specific DER schedule, i.e., for a specific nodal demand, $p_{j, t}$ and $q_{j, t}$, and root node voltage $v_{0, t}$.

Consider the DLMCs at node $n \in \mathcal{N}^{+}$, and time period $t$. It was shown in [1] that DLMCs can be decomposed into additive components that include: the cost at the substation, plus the real and reactive power marginal losses, plus the voltage and ampacity congestion, plus a new transformer marginal aging component. In this work, we supplement the latter component taking into account cable aging. In fact, following our analysis in the previous section, we show that the formulas derived for the transformers essentially carry over to the cables with an adjustment of the parameters. For brevity, we only provide PDLMCs, omitting voltage/ampacity congestion, as follows:

$$
\begin{aligned}
& \lambda_{n, t}^{P}=c_{t}^{P}[\text { Real Power Cost at Substation] } \\
& +c_{t}^{P} \sum_{j} r_{j} \frac{\partial l_{j, t}}{\partial p_{n, t}}+c_{t}^{Q} \sum_{j} x_{j} \frac{\partial l_{j, t}}{\partial p_{n, t}} \text { [Marginal Losses] } \\
& +\sum_{j \in \mathcal{N}_{X f}^{+} \cup \mathcal{N}_{C}^{+}} \pi_{j, t} \frac{\partial l_{j, t}}{\partial p_{n, t}},[\text { Marginal Aging] }
\end{aligned}
$$

where the sensitivities of the $\frac{\partial l_{j, t}}{\partial p_{n, t}}$ are calculated by the solution of a linear system derived from the power flow equations.

Before proceeding with the elaboration of parameter $\pi_{j, t}$, let us first consider the inter-temporal impact of aging that extends beyond the day-ahead, by extending the horizon to the next half day; the extended horizon is denoted by $T_{\text {ext }}$.

For transformer $j \in \mathcal{N}_{X f}^{+}, \pi_{j, t}$ is given by:

$$
\pi_{j, t}=\epsilon_{j}^{T O}\left[\sum_{t^{\prime}=t}^{T_{e x t}}\left(\frac{3}{4}\right)^{t^{\prime}-t} \tilde{\xi}_{j, t^{\prime}}^{H}\right]+\eta_{j}^{H} \tilde{\xi}_{j, t}^{H},
$$

where $\tilde{\xi}_{j, t}^{H}=\sum_{m} \xi_{j, t, m}^{H} a_{m}^{H}$, with $\xi_{j, t, m}^{H}$ the dual variable of constraint (10), and $\eta_{j}^{H}=\frac{0.8 \Delta \bar{\theta}_{j}^{H}}{l_{j}^{R}}$. The first part in (23) refers to the inter-temporal dynamics of the top-oil, which are discounted by a factor that is decreasing in time, while applying a higher weight on the high HST hours, and also includes the impact that extends beyond the optimization horizon. The second part refers to the winding contribution, which only affects the current time period. Notably, it should be $\sum_{m} \xi_{j, t, m}^{H}=c_{j}$; if only 
one constraint is binding then its dual $\xi_{j, t, m}^{H}$ should equal $c_{j}$. Hence, assuming that for a given $\theta_{j, t}^{H}$, the AAF slope is $\tilde{a}_{j, t}^{H}$, and using typical values in (23), we have:

$$
\pi_{j, t}=\frac{c_{j}}{l_{j}^{R}}\left[9.185 \sum_{t^{\prime}=t}^{T_{e x t}}\left(\frac{3}{4}\right)^{t^{\prime}-t} \tilde{a}_{j, t^{\prime}}^{H}+20 \tilde{a}_{j, t}^{H}\right],
$$

where the slope $\tilde{a}_{j, t}^{H}$ is given by:

$$
\tilde{a}_{j, t}^{H}=\frac{15000}{\left(273+\theta_{j, t}^{H}\right)^{2}} \exp \left(\frac{15000}{383}-\frac{15000}{273+\theta_{j, t}^{H}}\right) .
$$

For cable $j \in \mathcal{N}_{C}^{+}, \pi_{j, t}$ is given by:

$$
\pi_{j, t}=\epsilon_{j}^{C S}\left[\sum_{t^{\prime}=t}^{T_{e x t}}\left(\frac{2}{3}\right)^{t^{\prime}-t} \tilde{\xi}_{j, t^{\prime}}^{C}\right]+\eta_{j}^{C} \tilde{\xi}_{j, t}^{C},
$$

where $\tilde{\xi}_{j, t}^{C}=\sum_{m} \xi_{j, t, m}^{C} a_{m}^{C}$, with $\xi_{j, t, m}^{C}$ the dual variable of constraint (20), and $\eta_{j}^{C}=\frac{\Delta \bar{\theta}_{j}^{C}}{l_{j}^{R}}$. The first part in (23) refers to the inter-temporal dynamics of the cable surface, which are discounted by a factor that is decreasing in time, while applying a higher weight on the high conductor core temperature hours, and also includes the impact that extends beyond the optimization horizon. The second part refers to the conductor core contribution, which only affects the current time period. Notably, it should also be $\sum_{m} \xi_{j, t, m}^{C}=c_{j}$; if only one constraint is binding then its dual $\xi_{j, t, m}^{C}$ should equal $c_{j}$. Hence, assuming that for a given $\theta_{j, t}^{C}$, the AAF slope is $\tilde{a}_{j, t}^{C}$, and using typical values in (26), we have:

$$
\pi_{j, t}=\frac{c_{j}}{l_{j}^{R}}\left[12.667 \sum_{t^{\prime}=t}^{T_{e x t}}\left(\frac{2}{3}\right)^{t^{\prime}-t} \tilde{a}_{j, t^{\prime}}^{C}+40 \tilde{a}_{j, t}^{C}\right]
$$

where the slope $\tilde{a}_{j, t}^{C}$ is given by:

$$
\tilde{a}_{j, t}^{C}=\frac{B}{\left(273+\theta_{j, t}^{C}\right)^{2}} \exp \left(\frac{B}{363}-\frac{B}{273+\theta_{j, t}^{C}}\right) .
$$

Direct comparison of (23)-(25) with (26)-(28) shows that essentially the same formulas apply to both transformer and cables with an adjustment of parameters.

\section{Results}

We consider a $13.8 \mathrm{kV}$ distribution feeder of a municipal distribution feeder, Holyoke Gas and Electric, MA, US. The feeder serves a mix of commercial and residential loads, and includes 307 nodes, 110 transformers, and 30 cables. Feeder data are briefly presented in [1] and in greater detail in [9]. In this work, we are interested in estimating the aging DLMC components for specific load profiles.

Consider the P-DLMC aging component at leaf node $n$, hour $t$. It includes the aggregate impact of transformers and cables, i.e., of line $j \in \mathcal{N}_{X f}^{+} \cup \mathcal{N}_{C}^{+}$, which is proportional to the sensitivities $\frac{\partial l_{j, t}}{\partial p_{n, t}}$. Ignoring second order effects, the sensitivities of cables $j$ that are upstream node $n$, and the sensitivity of the service transformer that connects to node $n$, i.e., of $j=n$, can be

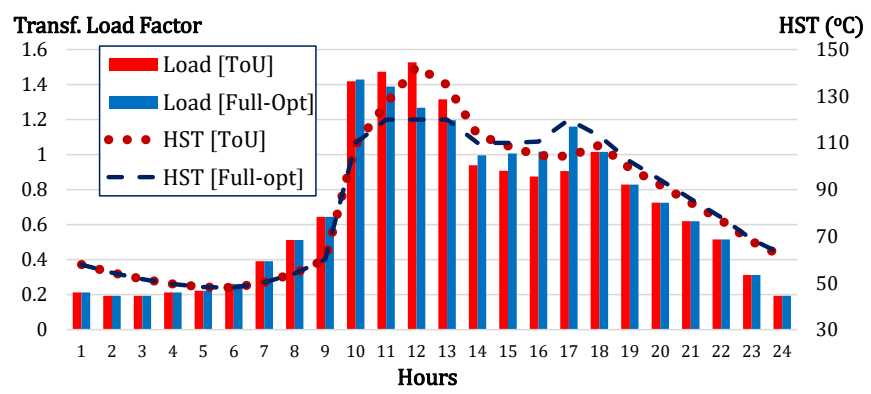

Fig. 1. Transformer Load Factor and HST.

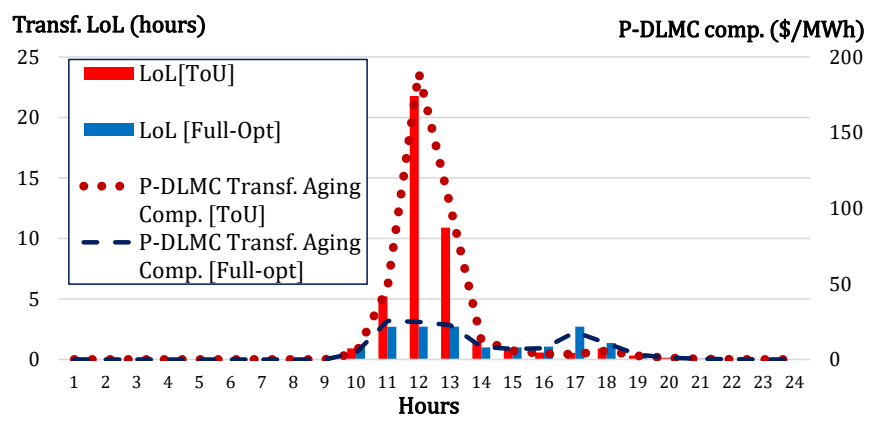

Fig. 2. Transformer LoL and P-DLMC Aging Component.

approximated by $\frac{\partial l_{j, t}}{\partial p_{n, t}}=\frac{\partial l_{j, t}}{\partial P_{j, t}} \frac{\partial P_{j, t}}{\partial p_{n, t}} \approx 2 P_{j, t}$ (where we considered p.u. values). The remaining sensitivities can be ignored. Hence, the P-DLMC aging component at a specific leaf node is affected by the aging of the transformer that serves this node (local impact), plus the aging of all upstream cables. Also, the real power flow can be approximated by $P_{j, t} \approx K_{j, t} S_{j}^{R} \cos \phi_{j, t}$, where $K_{j, t}$ is the load factor, $S_{j}^{R}$ the rated apparent power, and $\cos \phi_{j, t}$ the power factor; $l_{j}^{R}$ is also defined by $\left(S_{j}^{R}\right)^{2}$.

In Fig. 1, we present the load profiles and resulting HSTs for a 30-kVA transformer that serves a commercial node, in a summer day, with a 6-EV penetration, under two scheduling options. The first profile is the result of a ToU tariff, where the EVs adjust their profile according to the real power costs at the substation, $c_{t}^{P}$. The second option is the result of a centralized AC OPF enhanced to include transformer aging, referred to in [1] as Full-opt. The ToU HST profile exhibits a spike during hours $11-13$, reaching up to about $140^{\circ} \mathrm{C}$. Full-opt has a smoother load profile (load is shifted) resulting in a smoother HST profile, with a plateau of about $120^{\circ} \mathrm{C}$ during hours 11-13, and a small rise at the same level during hour 17. Fig. 2 shows the transformer LoL profiles and resulting estimated P-DLMC marginal aging component (considering a 0.9 power factor). ToU aggregate LoL reaches about 44 hours, whereas Full-opt about 17 hours. P-DLMCs follow the same shape with the LoL with a spike of about $190 \$ / \mathrm{MWh}$ under ToU, whereas Full-opt P-DLMCs reach up to $25 \$ / M W h$.

In Fig. 3, we present load profiles and resulting conductor core temperatures for a cable, with rated current $110 \mathrm{~A}$ (2.63MVA), an hourly cost of $0.2 \$$, at soil temperature $18^{\circ} \mathrm{C}$. The load profiles are proportional to the transformer profiles (adjusted with a factor of 0.85). Conductor core temperature reaches up to $130^{\circ} \mathrm{C}$ during hour 12 under ToU, whereas it 


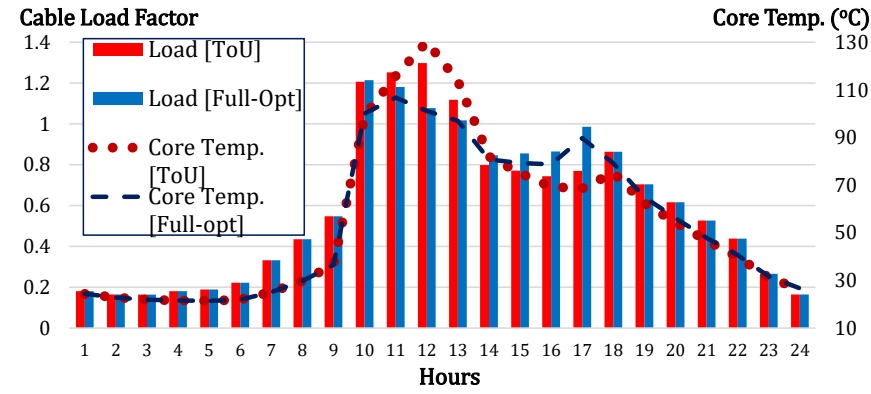

Fig. 3. Cable Load Factor and Core Temperature.

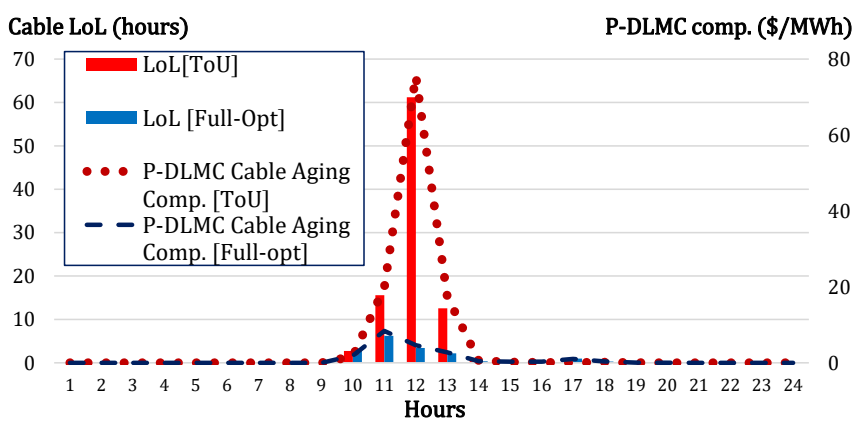

Fig. 4. Cable LoL and P-DLMC Aging Component.

reaches about $106^{\circ} \mathrm{C}$ under Full-opt, with a smaller rise at about $90^{\circ} \mathrm{C}$ during hour 17. Fig. 4 presents the LoL and P-DLMC profiles. The LoL is estimated for a value of $B=15000$. The cable power factor is also considered as 0.9. ToU aggregate LoL reaches about 93 hours, whereas Full-opt aggregate LoL reaches about 17 hours. Similarly to the transformers, P-DLMCs follow the same shape with the LoL with a spike of about 75\$/MWh under ToU, whereas Full-opt P-DLMCs reach up to $8 \$ / M W h$. Viewed as price signals, the P-DLMC spikes under ToU induce a shift of demand, thus leading to smoother profiles (Full-opt). We should note that the P-DLMCs are proportional to the cable hourly cost, $c_{j}$. Also, the impact of parameter $B$ is explained by the slope $\tilde{a}_{j, t}$ using (28). For instance a value of $B=10000$ would yield aggregate LoL of 31 (12) hours, ith a highest P-DLMC of about 13 (3) \$/MWh, under ToU (Full-opt). A cable hourly cost of $0.4 \$$ (e.g., a cable with double length) would result in double P-DLMC values.

\section{Conclusions}

The methodology proposed in this work leverages the flexibility of grid components, namely transformers and cables, to sustain overloads for short periods of time, by including the asset aging cost into active distribution network operational planning. Employing sensitivity analysis, we estimate distribution network marginal costs, and elaborate on the marginal aging DLMC components. The analysis illustrates the similarities between the transformer and the cable marginal aging components and the inter-temporal impacts of the respective thermal models. It also provides estimates of these components for given load profiles, highlighting their use as price signals that incentivize optimal DER scheduling.
The results show that "open-loop" pricing schemes, e.g., ToU, are likely to create significant overloading conditions, which in turn lead to increased aging of network infrastructure. DLMC spikes, when accounting for marginal aging, provide the correct signals to optimally shift loads (e.g., EV charging), should the latter be able to adapt their profiles to DLMCs (see e.g., Full-opt). The resulting much smoother profiles exemplify DLMCs as financial incentives that are key to optimize distribution network and DER operation.

In our future work, we will discuss the planning implications of spatiotemporally varying DLMCs, which also convey sufficient information considering DERs as non-wires solutions [10]. Furthermore, we will address cost considerations that relate with the risk of failure, which increases rapidly with aging, and extend our analysis to aging of overhead lines [11].

\section{Acknowledgements}

This research paper benefited from the support of the FMJH Program PGMO and from the EDF support to this program.

\section{References}

[1] Andrianesis, P., Caramanis, M.: 'Distribution Network Marginal Costs: Enhanced AC OPF Including Transformer Degradation', IEEE Trans. Smart Grid, 2020, 11, (5), pp. 3910-3920.

[2] IEEE Guide for Loading Mineral-Oil-Immersed Transformers, IEEE Standard C57.91, Mar. 7, 2012.

[3] Power Transformers, Part 7: Loading Guide for MineralOil-Immersed Power Transformers, BS IEC Standard 60076-7:2018, Jan. 1, 2018.

[4] IEC Standard 60287, Electric cables -- Calculation of the current rating, 2011.

[5] IEC Standard 60853, Calculation of the Cyclic and Emergency Current Ratings of Cables, 1989.

[6] Andrianesis, P., Caramanis, M.: 'Optimal Grid - Distributed Energy Resource coordination: Distribution Locational Marginal Costs and hierarchical decomposition', 57th Allerton Conf., Monticello, IL, 2019.

[7] Baran, M. E., Wu, F. F.: 'Optimal capacitor placement on radial distribution systems', IEEE Trans. Power Del., 1989, 4, (1), pp. 725-734.

[8] Farivar, M., Low, S. H.: 'Branch-flow model: Relaxations and convexification - Part I', IEEE Trans. Power Syst., 2013 28, (3), pp. 2554-2564.

[9] Andrianesis, P., Caramanis, M.: 'Distribution network marginal costs - Part II: Case study based numerical findings', 2019. [Online]. Available: arXiv:1906.01572.

[10] Andrianesis, P., Caramanis, M., Masiello, R.D., et al.: 'Locational Marginal Value of Distributed Energy Resources as Non-Wires Alternatives', IEEE Trans. Smart Grid, 2020, 11, (1), pp. 270-280.

[11] Dupin, R., Kariniotakis, G., Michiorri, A.: 'Overhead lines Dynamic Line rating based on probabilistic dayahead forecasting and risk assessment', Int. J. Electr. Power Energy Syst., 2019, 110, pp. 565-578. 